\title{
Dynamic state of water in excised Ligustrum lucidum branches observed by dedicated micro-magnetic resonance imaging
}

\author{
Hiromi Kano", Mika Koizumi ${ }^{2,}$, \\ ${ }^{1}$ Oak-Hill Georgic Patch-Work Laboratory, Chiba, Japan \\ ${ }^{2}$ Research Institute for Science and Engineering, Waseda University, Tokyo, Japan
}

Email address:

toradaiou@gamma.ocn.ne.jp (H. Kano),m.koizumi4@kurenai.waseda.jp (M. Koizumi)

\section{To cite this article:}

Hiromi Kano, Mika Koizumi. Dynamic State of Water in Excised Ligustrum Lucidum Branches Observed by Dedicated Micro-Magnetic Resonance Imaging. Plant. Vol. 2, No. 3, 2014, pp. 33-40. doi: 10.11648/j.plant.20140203.12

\begin{abstract}
The dynamic state of water was investigated for tree branches by a combined k-space and q-space imaging method using a dedicated magnetic resonance imaging (MRI) device equipped with a 1.0-T permanent magnet. Changes in the ${ }^{1} \mathrm{H}-\mathrm{NMR}$ image due to the application of pulsed-field gradients (PFG) of stepping magnitude were measured using a phantom consisting of flow water and stationary water; the relationship between the flow velocities and the diffusion coefficients of water was studied. The method was applied to excised Ligustrum lucidum branches under conditions with and

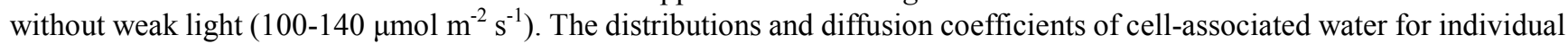
tissues in the branches were determined in relation to morphology. Large amounts of water existed but diffusion coefficients were not high in the cambium. Though the water amounts were moderate, the highest diffusion coefficient exceeded that for pure water in the secondary xylem. The conduit tubes were smaller than the imaging pixel, unlike the phantom, so the determined values might be perturbed by the conditions of the ambient cell-associated water. However, positional responses in the xylem flow to light were detected, and a flow velocity up to $0.71 \mathrm{~mm} \mathrm{~s}^{-1}$ by transpiration was recorded. The movement of water in the phloem was not distinguished from large amounts of water in adjacent tissues, probably due to the very small cells and slow rates of flow in the phloem.
\end{abstract}

Keywords: Dedicated MRI, Diffusion Coefficient, Flow-encoded Imaging, Ligustrum lucidum Branch, Morphology, Water State

\section{Introduction}

Long-distance transport of water between tissues is essential for organizing a plant body as a whole, which closely relates to transpiration, nutrient uptake, and materials transport, and thus profoundly concerns vegetation, and its response and adaptation to environmental changes. Water transport in a tree is driven by the vapor pressure difference between air and soil, and also by the internal osmotic pressure difference between tissues. Pressure probes have been applied to measure the water potential, and water or solute membrane permeability, either by diffusion or under hydrostatic pressure gradients [1-3] for parts of plants. Heat-tracer methods have been used to measure mass flow in the xylem of intact trees [4].

Stems and branches transport water consist of several differentiated tissues such as the epidermal tissue, the cortex, the phloem, the cambium, the outer xylem, and the inner xylem, with parenchyma cells and conduit tubes where water conditions vary according to the position, even within a branch, during the growth stage of a tree. In this regard, magnetic resonance imaging (MRI) is suitable for investigate the physical and dynamic state of water for individual tissues $[5,6]$. The fast-gradient echo-imaging method combined with a pulsed-field gradient (PFG) has been used for tracking the rapid responses of transpiration to on-and-off patterns of light [7, 8]. Another approach is the q-space imaging method, where the application of PFG is combined with the spin-echo two-dimensional Fourier transform (2D-FT) method [9, 10]. The flow velocity in vasculature and the diffusion rate of water in tissue cells are obtained simultaneously. This method had a weak point in that it took a rather long period to acquire a set of data. Recently, the measurement time was shortened considerably by using the turbo-spin echo method [11]. 
This paper reports the measurement of water state for a phantom and excised Ligustrum lucidum branches by a combined k-space and q-space imaging method. The repetition time (TR) for radio-frequency projection was shortened in order to obtain a set of data within the available time, and a dedicated MRI apparatus equipped with a permanent magnet was employed, which simplified the handling during the measurement procedure.

\section{Materials and Methods}

\subsection{Plant Materials}

The branches of Ligustrum lucidum were collected on May 26 and August 25, 2012, from the same tree on the campus of Waseda University. Branches grew actively between the two sampling times. The leaf-amount of the former was $162.4 \mathrm{~g}$ and that of the latter was $212.3 \mathrm{~g}$.

\subsection{Probing Water Molecules}

Dynamic NMR imaging was carried out by a combined k-space and q-space imaging method according to Callaghan [9]. The experiment was first tested using a phantom composed of flow water and stationary water, and the method was then applied to the excised Ligustrum lucidum branches.

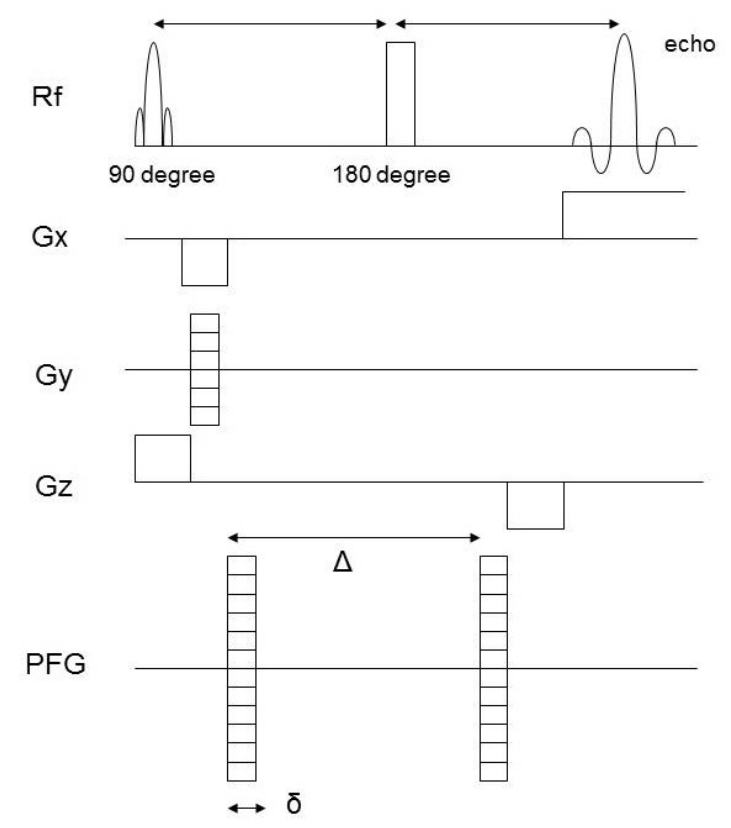

Fig. 1. Sequence of the combined $k$-space and $q$-space imaging method. The row for $R f$ indicates the timing of the projection of 90-degree and 180-degree radio-frequencies and generation of the echo-signal. Gx indicates the magnetic field gradients for read encoding; Gy for phase encoding; and $G z$ for determining slice thickness. The row for PFG indicates the application of pulsed-field gradients of $\delta \mathrm{ms}$ durations with a $\Delta$ ms separation.

The pulse sequence is presented in Fig. 1. The top line (Rf) indicates the timing of the radio-frequency projections and echo-signal generation; the second line (Gx) is the magnetic field gradient for read encoding; the third line (Gy) is for phase encoding; and the fourth line $(\mathrm{Gz})$ is for slicing an image plane. This is the sequence of the spin-echo 2D-FT (k-space imaging) method. The last line indicates the pulsed-field gradients (PFG). Pulses with the same magnitude are applied before and after the 180-degree radio-frequency pulse (top line; $\mathrm{Rf}$ ) with stepping intensity at each signal acquisitions, which becomes the flow-encoding gradients (q-space imaging). Symbol $\delta$ represents the duration of $\mathrm{PFG}$, and $\Delta$ represents the diffusion period. The method simultaneously probes the diffusion coefficient and the flow velocity of water molecules, the theory of which will be explained using the results for the phantom.

\subsection{MRI Apparatus and Measurements}

A dedicated MRI (Fig. 2A) equipped with a 1.0-Tesla (T: strength of a magnet) permanent magnet with a $60-\mathrm{mm}$ pole gap (a) (Neomax Engineering Co. Ltd., Gunma, Japan) and a spectrometer (b) based on a Windows personal computer (MRTechnology Inc., Tsukuba, Japan) [12] was used. The detector was made of a $30-\mathrm{mm}$ diameter solenoid coil and the measurement cell temperature was controlled at $28^{\circ} \mathrm{C}$.

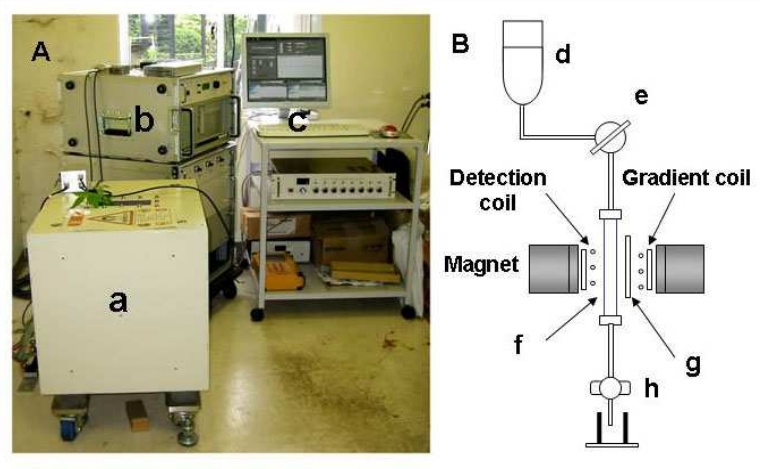

Fig. 2. Dedicated MRI apparatus (A), and measurement system for phantoms (B). (A) a. Permanent magnet with 1.0-T (42.58 MHz) field strength. b. MRI spectrometer using Windows on a personal computer. $c$. Console equipped with a liquid-crystal screen, a key-board, and a mouse. (B) d. Water reservoir. e. Stopcock for rough regulation. f. Flow phantom. $g$. Stationary phantom. $h$. Pinchcock for fine regulation of flow velocity.

Morphological images were acquired by the spin-echo 2D-FT method. TR was $1 \mathrm{~s}$ and the echo time (TE) was $7 \mathrm{~ms}$. Measurements were carried out with a $256 \times 128$ matrix and images were recreated on a $256 \times 256$ matrix. The resulting resolution was $117 \mu \mathrm{m}$.

Sixteen 2D-images were collected as a data set; a PFG pair was applied at each 2D image acquisition by changing the magnitude in steps from $-\mathrm{G}$ to $+\mathrm{G}$. TR was set at $1 \mathrm{~s}$, and it took $50 \mathrm{~min}$ to acquire a set of $2 \mathrm{D}$-image data. The duration of PFG application $(\delta)$ and the diffusion period $(\Delta)$ were essentially variable based on the property of the materials and the aims of the experiment; thus TE was also variable. Data were acquired with a $128 \times 64$ matrix and images were recreated on a $128 \times 128$ matrix. The resulting 
spatial resolution was $234 \mu \mathrm{m}$ with a $3.6-\mathrm{mm}$ slice thickness. Diffusion coefficient images were calculated using several 2D-images from the set of data. The series of image data applied with PFG were then compiled into a 3D-image datum and Fourier transformed after zero-filling to obtain a flow-encoded image.

Image analysis was conducted using the Image J program (a public domain JAVA image processing program (version $1.43 \mathrm{u})$ available on the Internet at http://rsb.info.nih.gov/ij/).

\subsection{System for Phantom}

Figure 2B depicts the measurement system for the phantom. Water was introduced from a water reservoir (d), through a cock (e), to a flow phantom of a $10-\mathrm{mm}$ diameter glass tube (f) in the detector of the MRI apparatus (Fig. 2Aa). A stationary phantom (g) of a 5-mm diameter glass tube was placed parallel to the flow phantom. Flowing water was exhausted to a measuring cup through a pinch cock (h) to finely adjust the flow rates. TE was $36.8 \mathrm{~ms}, \delta$ was $6 \mathrm{~ms}$ and $\Delta$ was $23 \mathrm{~ms}$.

\subsection{System for Branches}

The branch was trimmed and inserted into a measuring cup under the magnet through the detector of the MRI (Fig. 3A). Three light sources (p) holding three LED lamps of different light qualities were used. The light intensity was from 100 to $140 \mu \mathrm{mol} \mathrm{m} \mathrm{m}^{-2} \mathrm{~s}^{-1}$ on the centre leaves of the branch. Light stands made of wood, vinyl rope, and tapes enabled adjusting the geometry of the lamps to the shape of the branch (Fig. 3B). A dark room was made using black curtains. The amounts of water taken up were $10 \mathrm{ml}$ in the light and $4.5 \mathrm{ml}$ in the dark on May 26, and $27 \mathrm{ml}$ in the light and $10 \mathrm{ml}$ in the dark on August 25. TR was set at $1 \mathrm{~s}, \delta$ at 8 $\mathrm{ms}$, and $\Delta$ at $25 \mathrm{~ms}$; TE was thus $40.4 \mathrm{~ms}$.

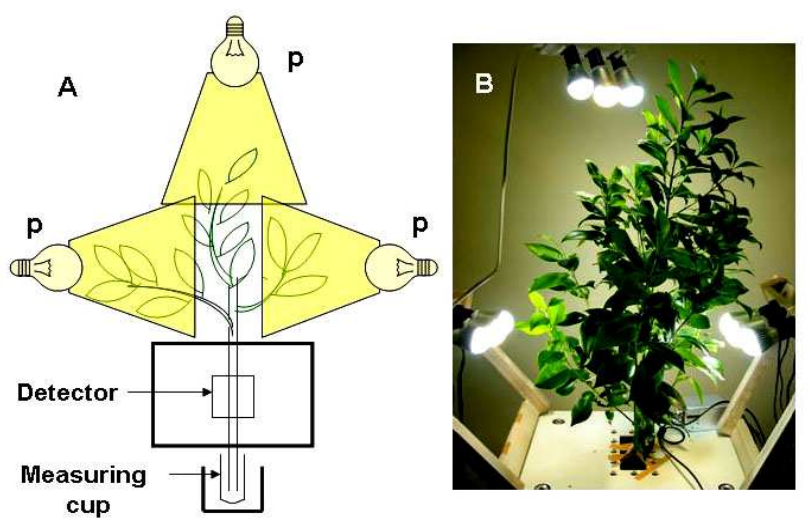

Fig. 3. Measurement system for tree branches $(A)$, and the light-projection system (B). (A) A tree branch was inserted into a measuring cup under a magnet through a detector coil, and the amount of absorbed water was measured. Light was provided by three projectors (p) consisting of three $L E D s$ with different light qualities. The small research room $(200 \mathrm{~cm} \times 500$ cm) was turned into a dark room by black curtains. (B) The positions of the light projectors were adjusted according to the shape and geometry of the branches using wood stands, vinyl ropes, and tape.

\section{Results}

\subsection{Experiments using the Phantom}

The images applied with PFG at $0 \mathrm{mT} \mathrm{m}^{-1}, 37 \mathrm{mT} \mathrm{m}^{-1}$ and $74 \mathrm{mT} \mathrm{m}^{-1}$ are presented in Fig. 4 (A-C). The large left tube is the flow phantom, and the small right tube is the stationary phantom. Since PFG acts as the square of the magnitude, the image at $74 \mathrm{mT} \mathrm{m}^{-1}$ (Fig. 4C) was very weak. The attenuation of the averaged signal intensity for the two tubes was plotted against the square of PFG on a logarithmic scale [13], which resulted in straight lines (Fig. 4D).

The diffusion coefficients can theoretically be obtained by Stejskal-Tanner's equation based on the plots; however, in the current investigation, calibration using pure water (the stationary phantom) is necessary. A short TR (1 s) is employed to reduce the measurement time, so this might cause signal saturation for high-mobility water. The slope of the plot for the stationary phantom (Fig. 4Da) corresponds to a diffusion coefficient of $2.3 \times 10^{-5} \mathrm{~cm}^{2} \mathrm{~s}^{-1}$ at $28^{\circ} \mathrm{C}$. Nor remarkable difference was observed in the plots between the stationary phantom (Fig. 4Da) and the flow phantom (Fig. $4 \mathrm{Db}$ ); the flow of water did not affect the diffusion. The directed movement of water molecules was potentially encoded on the images as follows.
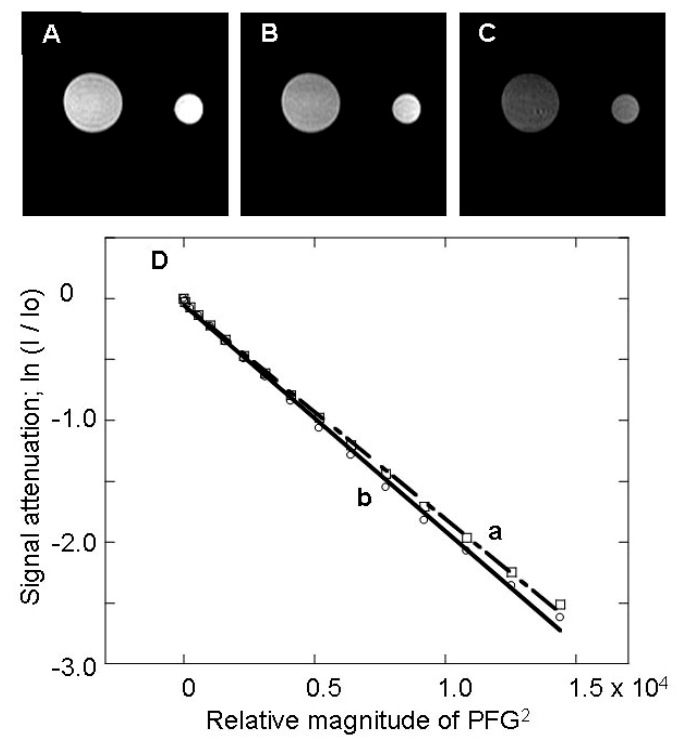

Fig. 4. Effects of pulsed-field gradients on the images of the phantom $(A-C)$ and logarithmic plots (D) of the signal attenuation for the stationary (a) and flow phantom (b). Images applied with PFG are presented. (A) $0 \mathrm{mT}$ $m^{-1}$. (B) $38 \mathrm{mT} \mathrm{m}^{-1}$. (C) $76 \mathrm{mT} \mathrm{m}{ }^{-1}$. The slope of the logarithmic plot for the stationary phantom (a) was $2.3 \times 10^{-5} \mathrm{~cm}^{2} \mathrm{~s}^{-1}$ at $28^{\circ} \mathrm{C}$, the diffusion coefficient of pure water.

The 3D-image datum made by compiling a series of 2D-image data applied with stepping PFG (Fig. 4) was Fourier transformed after zero-filling to construct a tertiary image, where the first axis represents the $\mathrm{X}$-axis and the second represents the $\mathrm{Y}$-axis in physical space ( $\mathrm{k}$-space), and the Z-axis corresponds to the flow-encoded space (q-space). Figure 5A depicts the distribution of flow-encoded spins in 
the $\mathrm{X}-\mathrm{Y}$ plane at the $64^{\text {th }}$ of 128 slices in the $\mathrm{Z}$-axis direction. Figure $5 \mathrm{~B}$ represents the distribution of spins on the $\mathrm{Y}-\mathrm{Z}$ plane at the central line (arrow a) on the flow phantom in Fig. 5A. The signals thus indicate the amount of the spins migrated in q-space. The q-space signals in the flow tube shifted more to the right as the position approached its centre, while those in the stationary tube were not disturbed. As a result, signal intensities around the centre of the flow tube in the $64^{\text {th }} \mathrm{X}-\mathrm{Y}$ slice were weak (Fig. $5 \mathrm{~A}$ ), which is referred to as dynamic displacement [9].

Displacement was right as the movement of water molecules was downward in the current experiments. Dynamic displacement profiles at line (b) for the stationary tube and at line (c) for the flow tube in Fig. 5B are presented in Fig. 5C. The distance between $\alpha$ (the peak position for stationary water) and $\beta$ (the peak position for flow water) corresponds to the rate of water movement.
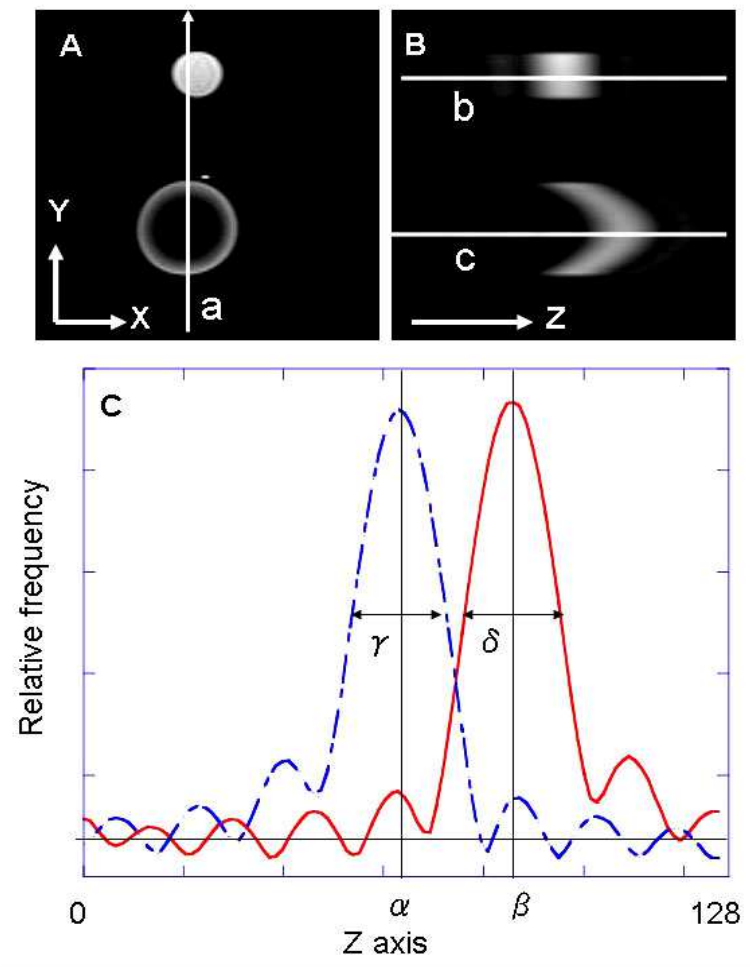

Fig. 5. Flow-encoded 3D-image constructed by using a series of 2D-images applied with PFG as seen in Fig. 4. (A) Image of actual (X-Y) space at the $64^{\text {th }}$ of 128 slices in $q$-space. The top circle is the stationary phantom, and the bottom one is the flow phantom. (B) Image of actual space (Y) and $q$-space $(Z)$ slice at the position indicated by line a in image $A$. (C) Dynamic displacement profiles of the stationary phantom (dashed line) at line $b$ and the flow phantom (solid line) at line c in image B. Peak $\alpha$ is positioned at 64 , and peak $\beta$ indicates the dynamic displacement of the flowing water. Full-width at half-maximum ( $\gamma$ and $\delta$ ) corresponds to the theoretical diffusion coefficients. However, in the current investigation, calibration was necessary to obtain the diffusion coefficients using Fig. 4.

Figure $6 \mathrm{~A}$ is an image of the flow rate based on the result in Fig. 5, with the signal intensity corresponding to the velocity. Figure $6 \mathrm{~B}$ is a surface plot drawing of Fig. 6A, the Z-axis of which indicates downward flow (upward movement of water is omitted).
Displacement shift of distances from $\alpha$ to $\beta$ (Fig. 5C) were plotted against the flow velocity of water measured by a cylinder cup (Fig. 7). A linear relation was obtained, which was assumed as a calibration curve. The shift was 0.21 versus $1 \mathrm{~mm} \mathrm{~min}^{-1}$ of flowing water for PFG with $\Delta=23 \mathrm{~ms}$ and $\delta=6 \mathrm{~ms}$, used for the experiments on the phantom, while the shift was 0.35 for PFG with $\Delta=25 \mathrm{~ms}$ and $\delta=8 \mathrm{~ms}$, used for the tree branches.

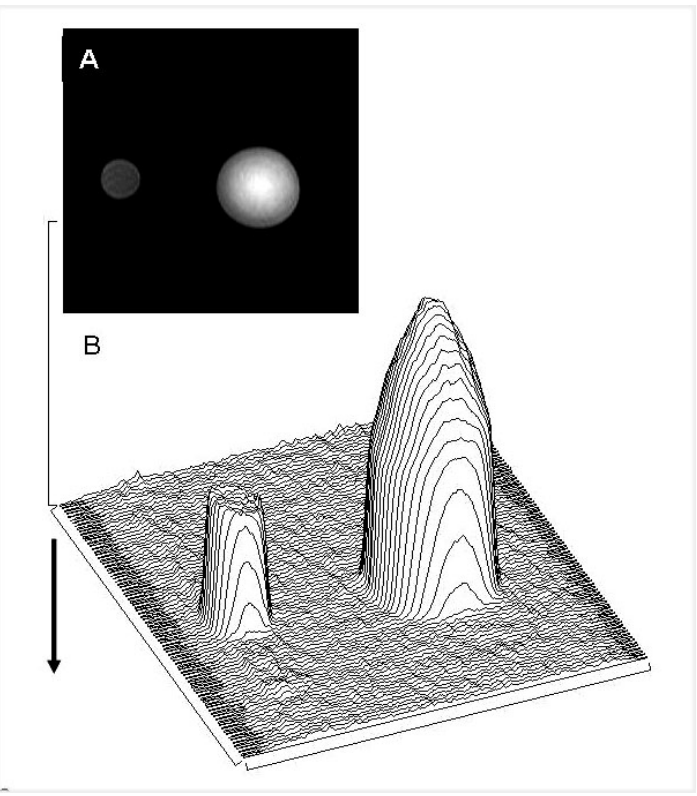

Fig. 6. Images showing the velocity of water movement (A) and its surface plot (B). (A) The small circle on the left indicates the movement of water by diffusion, and the large circle on the right indicates the velocity of the flowing water, calculated based on Fig. 5. (B) The arrow on the left side indicates upward, therefore, the water is flowing downward. Upward movement of the water is omitted.

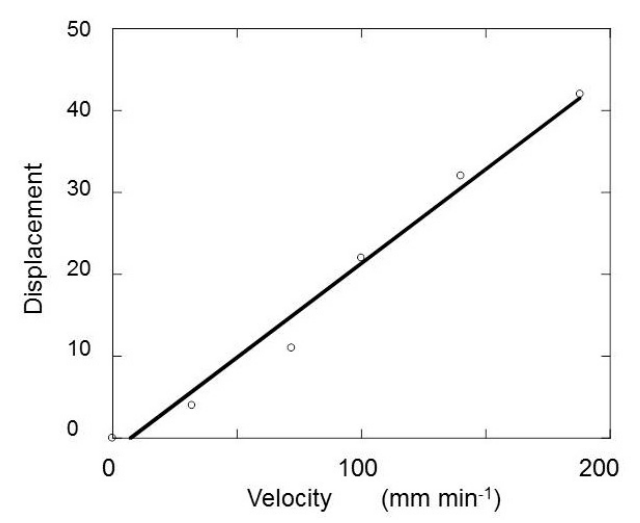

Fig. 7. Calibration curve for water flow: the relationship between the flow velocity of water and a shift of the peak in dynamic displacement profiles. Measurements were conducted at $\delta=6 \mathrm{~ms}, \Delta=23 \mathrm{~ms}$, and $T E=36.8 \mathrm{~ms}$.

\subsection{Distribution and Diffusion of Cell-associated Water for Tree Branches}

Cross-sections of a young branch $8 \mathrm{~mm}$ in outer diameter were stained with toluidine blue and observed by an optical microscope (Fig. 8). The outermost tissue was the cortex of the epidermal layer (a) containing air spaces, the phloem 
was outside the cambium (b), and the secondary xylem was situated inside (c). Considerable number of small conduit cavities were gathered just inside the cambium, radial strands passed through to the epidermal layer, and several irregular rows of conduit cavities were placed at right angles with the strands with approximately $250-\mu \mathrm{m}$ separations in the xylem (Fig. 8B). Ligustrum lucidum had concentric circles of conduits. The conduit cavities were approximately $50 \mu \mathrm{m}$, and the remained part of the secondary xylem was filled with smaller parenchyma cells between the strands (Fig. 8C).

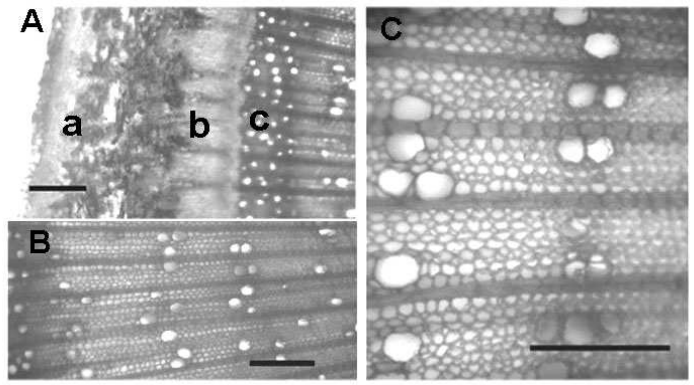

Fig. 8. Optical micrographs of an $8 \mathrm{~mm}$ diameter Ligustrum lucidum branch. (A) a. The epidermal layer. $b$. the cambium. $c$. the secondary xylem. (B) The secondary xylem. (C) The conduit cavities. Scale bars are $250 \mu \mathrm{m}$.
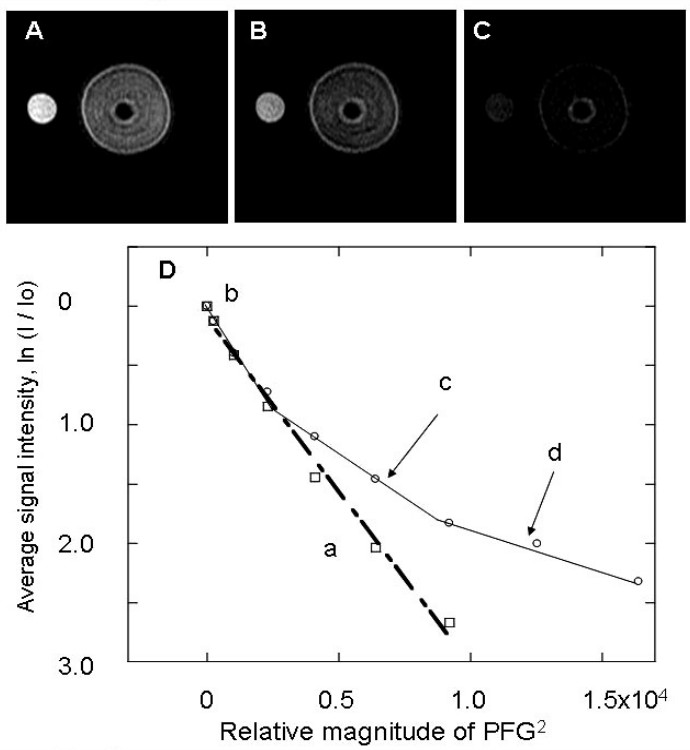

Fig. 9. Effects of pulsed field gradients on the images of a tree branch $(A-C)$ on August 25, and logarithmic plots (D) of signal attenuation for the stationary phantom (a) and the branch ( $b$ - $d$ ). Images with PFG applied are presented. (A) $0 \mathrm{mT} \mathrm{m}^{-1}$. (B) $38 \mathrm{mT} \mathrm{m}^{-1}$. (C) $76 \mathrm{mT} \mathrm{m}^{-1}$. The slope of the logarithmic plot of the stationary standard (a) is $2.3 \times 10^{-5} \mathrm{~cm}^{2} \mathrm{~s}^{-1}$ at $28^{\circ} \mathrm{C}$, the diffusion coefficient of pure water.

Images applied with PFG at $0 \mathrm{mT} \mathrm{m}^{-1}, 38 \mathrm{mT} \mathrm{m}^{-1}$ and 76 $\mathrm{mT} \mathrm{m}^{-1}$ to the branch on August 25 are presented in Fig. 9 (A-C). The averaged signal intensity of the branch and the stationary standard was plotted against the square of PFG on a logarithmic scale (Fig. 9D) [14, 15]. The plot for the stationary standard was a straight line like that in Fig. 4, the slope of which indicates the diffusion coefficient of pure water $\left(2.3 \times 10^{-5} \mathrm{~cm}^{2} \mathrm{~s}^{-1}\right)$. The effect of PFG was approximately 1.65 times stronger than in Fig. 4 because of the longer $\delta$ and $\Delta$. The plot for the tree broke into three lines; the first line $\left(2.7 \times 10^{-5} \mathrm{~cm}^{2} \mathrm{~s}^{-1}\right)$ was steeper than stationary water, the second was less steep $\left(1.2 \times 10^{-5} \mathrm{~cm}^{2} \mathrm{~s}^{-1}\right)$, and the third was almost flat $\left(0.5 \times 10^{-5} \mathrm{~cm}^{2} \mathrm{~s}^{-1}\right)$.

The ${ }^{1} \mathrm{H}-\mathrm{NMR}$ images represent the distribution of water in the branches on May 26 (Fig. 10A) and August 25 (Fig. 10B). The signals were weak in the epidermal layer (a), strong in the cambium including the phloem (b), and moderate in the secondary xylem (c). Weak signal areas were observed at the upper right and left side. In addition, there were arcs in the secondary xylem on May 26 (Fig. $10 \mathrm{~A}$ ), while signals in the secondary xylem were rather uniform except for thin weak concentric circles on August 25 (Fig. 10B). The signals of the primary xylem (d) were rather strong but the pith (e) had no signal.
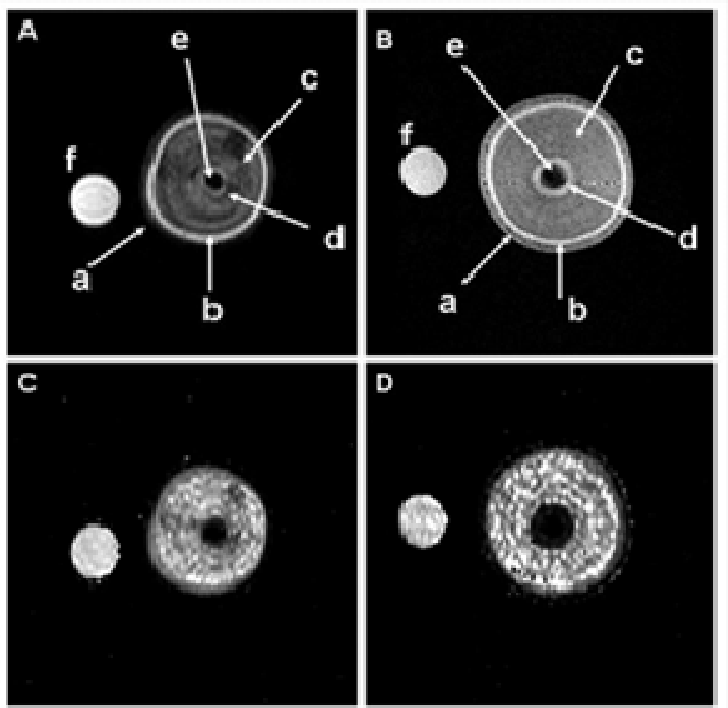

Fig. 10. ${ }^{1} H-N M R$ images of tree branches showing morphology and the corresponding diffusion coefficient images for May 26 ( $A$ and $C$ ) and August 25 (B and D). (A) and (B) a. Epidermal layer. b. Cambium. c. Secondary xylem. d. Primary xylem. e. Pith. f. Stationary phantom. (C) and (D) Diffusion coefficient images calculated using ImageJ.

Diffusion-coefficient images were calculated for the branch on May 26 in Fig 10C and on August 25 in Fig. 10D. The diffusion coefficient of the cortex tissue of epidermal layer (a) was null, and the values were low in the phloem and the cambium (b). High-value signals formed discontinuous concentric circles, and the remaining parts were low in the secondary xylem (c). The primary xylem (d) exhibited low values, and the pith was dark (e). The tendencies were similar for the two branches. The effect of light, though not marked, was observed along the concentric circles of conduits.

\subsection{Flow-encoded Images and the Velocity of Transpiring Water in Conduit Cavities}

Flow-encoded images are presented in Fig. 11 under light (A) and in the dark (B) for the branch of August 25. Signals 
were restricted to the secondary xylem, and dynamic displacement profiles were obtained in the light (C) and in the dark (D) at a position exhibiting strong signals (indicated by arrows). The image pixel $(234 \mu \mathrm{m})$ was so much larger than the diameter of the conduit tubes that signals from the conduits overlapped those of the ambient cell-associated water moving in Brownian motion. Consequently, the profiles of the flowing spins formed shoulders on the left side of the main diffusion peaks (Fig. 11C, D). Since spins due to Brownian motion exhibit symmetrical Gaussian-broadening (blue broken lines), the population of flow-encoded spins can be obtained by subtracting the spin population after the peak from that before the peak (green dotted lines in Fig. 11(Ca, Db)).

In the same way, we have:

Flow-encoded image $=$ Sum slices before Pmax - Sum slices after Pmax,

where Pmax is the slice position with the maximum spin population. The flow-encoded image (Fig. 11A, B) indicates the population of flowing spins. The dynamic displacement shift was to the left side (Fig. 11C, D), indicating that water
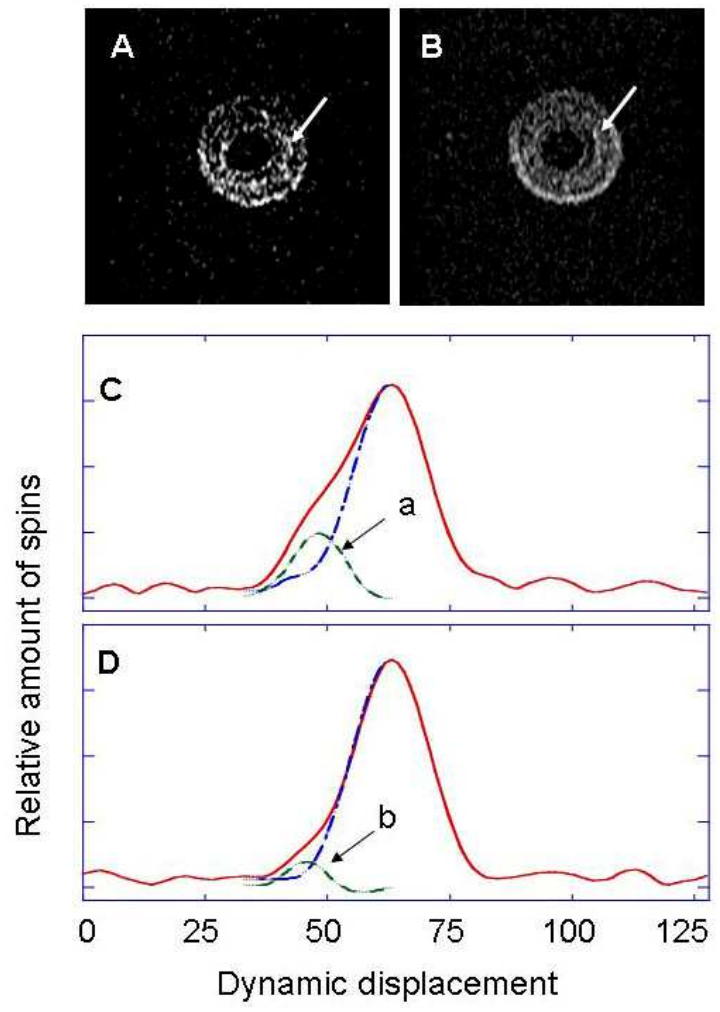

Fig. 11. Flow-encoded images (A and $B)$ and dynamic displacement profiles (C and D) for the branch on August 25. (A) and (C) In light. (B) and (D) In dark. Flow-encoded image were calculated by (Sum of slices before the peak) - (Sum of slices after the peak) in light (A) and dark (B) conditions. Slices $39^{\text {th }}$ through $64^{\text {th }}$ were taken for the former while slices $64^{\text {th }}$ through $89^{\text {th }}$ were taken for the latter. Dynamic displacement profiles (solid lines) in $q$-space at the positions indicated by arrows in $(A)$ and $(B)$ are presented in (C) and (D). The displacement shifts to the left, so the flow direction is upward. The right-side curves from the peak top were reversed (indicated by dotted lines) then subtracted from the left-side curves to obtain the dynamic displacements of the flow water molecules (dashed lines). molecules moved upward. The flow rate was calculated to be $0.71 \pm 0.10 \mathrm{~mm} \mathrm{~s}^{-1}$ at several positions with intense signals under light conditions (Fig. 11A). The velocity in the dark (Fig. 11D) was almost the same as that in the light (Fig. $11 \mathrm{C}$ ), but the number of flowing spins declined to $40 \%$ of the number in the light. This reduction accounted for decreased water uptake: $27 \mathrm{ml} \mathrm{h}^{-1}$ in the light, but $10 \mathrm{ml} \mathrm{h}^{-1}$ in the dark on August 25.

Light-enhanced images were calculated by subtracting the flow-encoded image in the dark (e.g., Fig. 11B) from that in the light (e.g., Fig. 11A). Figure 12A is for the branch on May 26, and Fig. 12C is for August 25. The images in Fig. $12(\mathrm{~B}, \mathrm{D})$ are superposition of the light-enhanced images on the corresponding ${ }^{1} \mathrm{H}-\mathrm{NMR}$ images, representing tissues morphology (Fig. 10A, B). Enhancements occurred in the secondary xylem forming arcs or discontinuous concentric circles, which seemed to coincide with the arrangement of conduit cavities (Fig. 8). Downward movement of the water was not obvious, and the phloem flow could not be distinguished from large amounts of water in adjacent tissues, probably because the cells were much smaller and the flow rates were slower in the phloem than in the conduits.
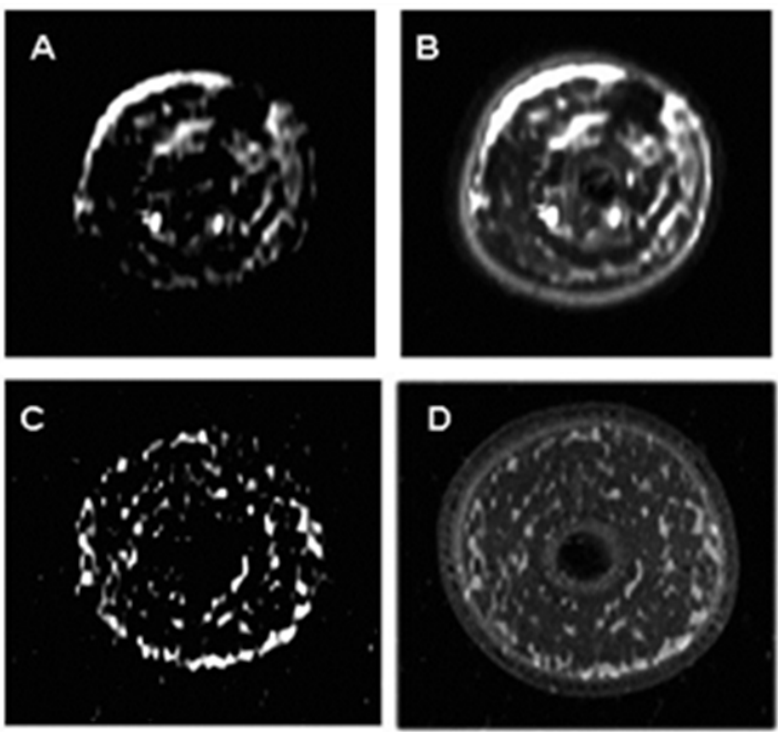

Fig. 12. Light-enhanced flow images for the tree branches for May 26 (A and $B$ ) and August 25 (C and D). (A) and (C) Light-enhanced flow images were obtained by subtracting the flow encoded image in the dark (e.g., Fig. 11B) from that in the light (e.g., Fig. 11A). (B) and (D) Light-enhanced flow images were super-positioned on the ${ }^{1} \mathrm{H}-\mathrm{NMR}$ image (Fig. 10A, B), revealing morphology.

\section{Discussion}

Specialized equipment has been devised to study water transport in xylem and phloem by MRI to examine intact potted plants [5, 6, 16-18]. In the current investigation, the transpiration of water, and the distribution and diffusion coefficients of cell-associated water were examined for excised Ligustrum lucidum branches in relation to morphology using a small MRI designed for common use. 
Theoretical explanations of diffusion-weighted images and flow-encoded images (Fig. 1) are made based on the results of phantom experiments (Figs. 4-7). To obtain a set of flow-encoded image data within an appropriate time (within $1 \mathrm{~h})$, a short repetition time $(\mathrm{TR}=1 \mathrm{~s})$ was employed, and thus theoretical diffusion coefficients [13] and flow velocities [9] could not be obtained; calibrations using standard phantoms were needed. In this regard, this study is the technical application of a combined k-space and q-space imaging method for tree branches at different growth stages and different seasons.

${ }^{1} \mathrm{H}-\mathrm{NMR}$ images (Fig. 10A, B) representing water distribution indicate the matrix size of metabolism, diffusion-coefficient images (Fig. 10C, D) indicate the metabolic rate in cells [19], and flow-encoded images (Figs. 11 and 12) indicate long-distance or inter-cellular transport of water. Water distribution patterns were not identical for the branches between early summer on May 26 and late summer on August 25; possibly due to the difference in growth stage, or anatomy, i.e., a near-branching position for the former (Fig. 10A) or non-branching position for the latter (Fig. 10B). The tendencies in the diffusion and flow of water were essentially similar. The highest diffusion coefficient exceeded that of a stationary standard of pure water (Fig. 9D). The movement of cell-associated water is considered to be elevated by metabolism; therefore, the diffusion coefficient images (Fig. 10C, D) indicate the fluctuations of cell activity in different places. Water amounts were large in the cambium, but metabolism was not so active and the epidermal tissues with a small amount of water seemed inactive. High diffusion coefficients formed discontinuous concentric circles in the secondary xylem where the water was moderate. The values for the primary xylem were low even though the signals in ${ }^{1} \mathrm{H}-\mathrm{NMR}$ were rather high, indicating that the tissue is not metabolically active.

Upward water movement was detected in the dark (Fig. 11B), and signal enhancement in the light revealed discontinuous concentric circles due to increased transpiration (Fig. 11A). The positions responding to light in the flow-encoded images (Fig. 12A, C) seem to coincide with areas where diffusion coefficients were high (Fig. 10C, D). Hence, the parenchyma cells around the conduit tubes in the secondary xylem (Fig. 8) are involved in transpiration. This finding supports the hypothesis that water flow within the xylem is maintained by internally circulated water between the phloem and xylem [17].

The flow velocity at several places or areas exhibiting intense flow signals in Fig. 11 (A, B) was calculated to be $0.71 \pm 0.10 \mathrm{~mm} \mathrm{~s}^{-1}$ based on dynamic displacement profiles, though there remains uncertainty due to larger pixels than for the conduits (Fig.8) [16]. The value is much smaller than the flow velocity reported for tomato $\left(3.1 \mathrm{~mm} \mathrm{~s}^{-1}\right.$ or $5.1 \mathrm{~mm}$ $\left.\mathrm{s}^{-1}\right)[11,20]$, caster bean $\left(3.7 \mathrm{~mm} \mathrm{~s}^{-1}\right)$, and tobacco $(1.8 \mathrm{~mm}$ $\left.\mathrm{s}^{-1}\right)$ among the herbs, about half that of poplar trees $(1.6 \mathrm{~mm}$ $\left.\mathrm{s}^{-1}\right)$ [20], but similar to caster bean seedlings $\left(0.5 \mathrm{~mm} \mathrm{~s}^{-1}\right)$ [16, 17]. Regrettably, the phloem could not be distinguished from adjacent cell-associated water, probably due to the slow flow rate [20] or the dimensions of phloem cells, which are several times smaller than conduit cells [6].

Since the excised branches are not regulated by a root system, the results do not represent the features of the long-distance transport of water in a plant functioning as an organic whole. However, the dynamic NMR imaging method using a small, light MRI with a permanent magnet (Figs. 2 and 3) facilitates studying the functions of differentiated tissues within branches and stems regarding the long-distance transport of water in response to internal and environmental changes [6].

\section{Reference}

[1] M.T. Tyree, S. Patino, J. Bennink and J. Alexander, "Dynamic measurements of root hydraulic conductance using a high-pressure flowmeter in the laboratory and field." Journal of Experimental. Botany, 1995. 46: 83-94.

[2] A.D. Tomos and R.A. Leigh, "The pressure probe: a versatile tool in plant cell physiology." Annual Review of Plant Physiology and Plant Molecular Biology, 1999. 50: 447-472.

[3] T. Henzler, R.N. Waterhouse, A.J. Smyth, M. Carvajal, D.T. Cooke, A.R. Schäffner, E. Steudle and D.T. Clarkson, "Diurnal variations in hydraulic conductivity and root pressure can be correlated with the expression of putative aquaporins in the roots of Lotus japonicus." Planta, 1999. 210: 50-60.

[4] D.M. Smith and S.J. Allen, "Measurement of sap flow in plant stems." Journal of Experimental Botany, 1996. 47: $1833-1844$.

[5] H. Van As, "Intact plant MRI for the study of cell water relations, membrane permeability, cell-to-cell and long-distance water transport." Journal of Experimental Botany, 2007. 58: 743-756.

[6] H. Van As and J. van Duynhoven, "MRI of plants and foods." Journal of Magnetic Resonance, 2013. 229: 25-34.

[7] E. Kuchenbrod, M. Landeck, F. Thürmer, A. Haase and U. Zimmermann, "Measurement of water flow in the xylem vessels of intact maize plants using flow-sensitive NMR imaging." Botanica Acta, 1996. 109: 184-186.

[8] M. Rokitta, U. Zimmermann and A. Haase, "Fast NMR flow measurements in plants using FLASH imaging." Journal of Magnetic Resonance, 1999. 137: 29-32.

[9] P.T. Callaghan, "Principles of nuclear magnetic resonance microscopy." 1991. Oxford Clarendon Press

[10] Y. Xia, V. Sarafis, E.O. Campbell and P.T. Callaghan, "Non invasive imaging of water flow in plants by NMR microscopy." Protoplasma, 1993.173: 170-176.

[11] T.W.J. Scheenen, D. van Dusschoten, P.A. de Jager and H. Van As, "Microscopic displacement imaging with pulsed field gradient turbo spin-echo NMR." Journal of Magnetic Resonance, 2000. 142: 207-215.

[12] M. Koizumi, S. Naito, N. Ishida, T. Haishi and H. Kano, "A dedicated MRI for food science and agriculture." Food Science and Technology Research, 2008. 14: 74-82. 
[13] E.O. Stejskal and J.E. Tanner, "Spin diffusion measurements: spin echoes in the presence of a time-dependent field gradient." The Journal of Chemical Physics, 1965. 42:288-292.

[14] C.D. Eccles, P.T. Callaghan and C.F. Jenner, "Measurement of the self-diffusion coefficient of water as a function of position in wheat grain using nuclear magnetic resonance imaging." Biophysical Journal, 1988. 53: 77-81.

[15] N. Ishida, H. Ogawa and H. Kano, "Diffusion of cell-associated water in ripening barley seeds." Magnetic Resonance Imaging, 1995. 13:745-751.

[16] P.T. Callaghan, W. Köckenberger and J.M. Pope, "Use of difference propagators for imaging of capillary flow in the presence of stationary fluid.” Journal of Magnetic Resonance, Series B, 1994. 104: 183-188.

[17] W. Köckenberger, J.M. Pope, Y. Xia, K.R. Jeffrey, E. Komor and P.T. Callaghan, "A non-invasive measurement of phloem and xylem water flow in castor bean seedlings by nuclear magnetic resonance microimaging." Planta, 1997. 201: 53-63.

[18] C.W. Windt, E. Gerkema and H. Van As, "Most water in the tomato truss is imported through the xylem, not the phloem: a nuclear magnetic resonance flow imaging study." Plant Physiology, 2009. 151:830-842.

[19] N. Ishida, M. Koizumi and H. Kano, "The NMR microscope: a unique and promising tool for plant science." Annals of Botany, 2000. 86: 259-278.

[20] C.W. Windt, F.J. Vergeldt, P.A. de Jager and H. Van As, "MRI of long-distance water transport: a comparison of the phloem and xylem flow characteristics and dynamics in poplar, castor bean, tomato and tobacco." Plant, Cell and Environment, 2006. 29: 1715-1729. 